\title{
INTERVIEW
}

For reprint orders, please contact: reprints@futuremedicine.com

\section{Molecular neuro-oncology: a forward-looking perspective}

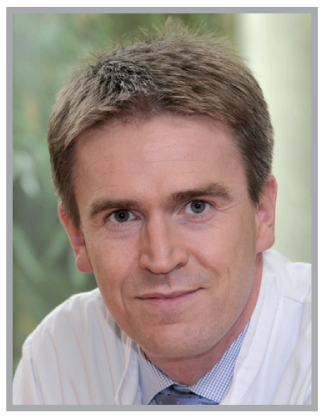

Professor Wolfgang Wick* speaks to Tess O'Neill, Head of Commissioning: Wolfgang Wick is the Chairman of the Department of Neuro-Oncology, Hertie Professor of Neuro-Oncology and Director at the National Tumor Center at the University of Heidelberg, Germany. $\mathrm{He}$ is conducting multicenter Phase III randomized trials for the Neuro-Oncology Working Group of the German Cancer Society, the European Organisation for Research and Treatment of Cancer as well as a number of multicenter trials with the pharmaceutical industry. He is a steering committee member of the Neuro-Oncology Working Group and the European Association for Neuro-oncology as well as chairman of the European Organisation for Research and Treatment of Cancer Brain Tumor Group. His main scientific interests include migration and invasion of glioma cells, biomarkers and radiosensitization.

How did your research interests come to focus on neuro-oncology \& which colleagues have been the most influential on your career so far?

I did my doctoral thesis in brain metastasis. I looked for genetic abnormalities in brain metastasis versus solid cancer in the body. This developed my interest in the field and I then furthered that by working in neuropathology. I was then based in neuro-oncology for a year at Harvard Medical School in Boston (MA, USA) and got really affected by the work I did there, which led to the later focus of my career.

In terms of influencing my career, Fred Hochberg at Harvard Medical School was my supervisor in my first very relevant practical year. One of the most influential and important neurooncologists is Michael Weller, with whom I did my training in Neurology in Tübingen (Germany).

Continuing efforts are being made to understand the role of molecular markers as predictors of outcome in gliomas. Which of these markers do you think should become part of routine clinical practice \& what are the barriers to achieving this?

I think for grade III tumors and probably also for grade II gliomas it is necessary to look for the $1 \mathrm{p} / 19 \mathrm{q}$ codeletion. Its also necessary to check for $I D H$ mutations because this will determine if it the outlook is generally favorable for the patient. If $I D H$ is not mutated we will have to check for MGMT. These are the three markers that are important for the general glioblastoma population. At the moment, in general glioblastoma, we have no marker to be incorporated into clinical routine. For the elderly patient, I would say that MGMT is also necessary to tell us which patients would not get the relevant benefits from temozolomide plus radiotherapy.

As the global population ages, effective treatment for older patients is
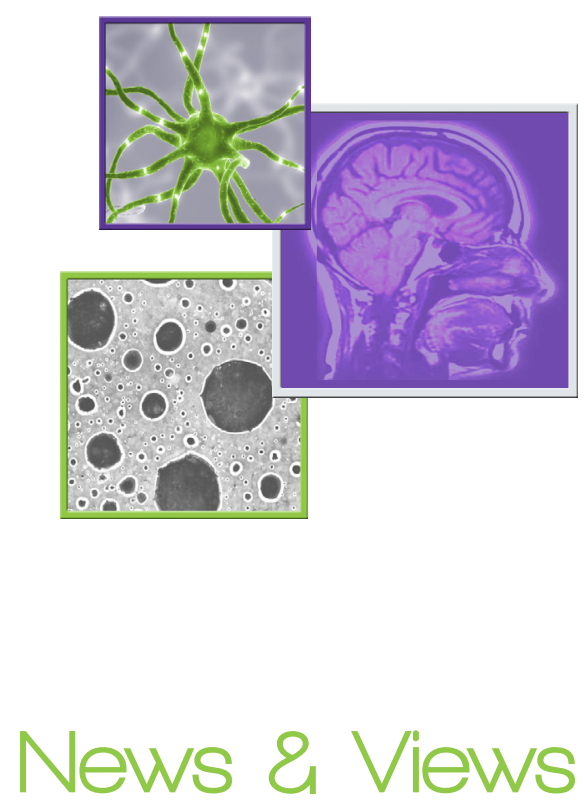

News

Journal Watch

Interview

*Abteilung Neuroonkologie, Neurologische Klinik \& Nationales Tumorzentrum, Universitätsklinik Heidelberg, Im Neuenheimer Feld 400, 69120, Heidelberg, Germany and KKE Neuroonkologie (G370), Deutsches Krebsforschungszentrum, Im Neuenheimer Feld 280, D-69120 Heidelberg, Germany; wolfgang.wick@med.uni-heidelberg.de

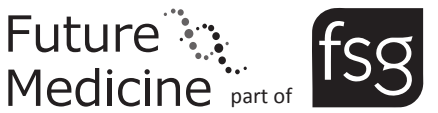


becoming a key focus in oncology. You recently reported on the difficulty of finding prognostic markers for elderly patients with gliomas. What are the next steps in this area of research?

I think there are three key areas of future research. The first is to focus on what the standard of care should be for patients with a methylated MGMT promoter. For instance, is it chemotherapy alone? With temozolomide? Is it radiochemotherapy? Do we need upfront radiotherapy? Is it of benefit to that patient group?

The second focus area should be to establish the role of more intensive treatment. For example, antiangiogenic treatment, but also other treatments in addition to upfront radiotherapy or radiochemotherapy or temozolomide chemotherapy, because the prognosis for these elderly patients is still extremely poor. First-line treatment is very important for these patients as they often don't make it to second-line treatments as they get too ill or show too much of a decline in their general health, regardless of whether they want to have therapy or not.

The third area is to really understand what makes elderly patients with a tumor different from younger patients. Is it just age? Is it just comorbidity? Or are there some molecular differences in the tumors? We need to ask whether the tumors are really the same across all ages.

Methylation studies have been carried out indicating that there may be less favorable prognostic factors in those elderly patients compared with the younger ones. But we need to make this information more established through further study. We need specific molecular markers for the designation of an elderly patient's tumor versus a nonelderly tumor.

\section{Q At the National Center for Tumor} Diseases the neuro-oncology department collaborates with hematology \& radiation oncology. What insights do you think this has provided \& how important do you think sharing ideas between different specialties will prove to be in advancing the field of oncology?

I think its very important to interact with the radiation department because radiotherapy is, for most brain tumors, one of the most important methods of therapy. Also, since most of us are neurologists in the NCT, we are of course also interested in any form of neurocognitive or functional side effects of any of the treatments.

From hemato-oncology we have seen how they have managed to subclassify their tumors into molecular categories - so they are more advanced than we are in that respect. For example, there are now $B R A F-$ mutated tumors and there are $A L K$-mutated tumors; there are all sorts of molecular markers defining tumor subgroups that we do not have to the same extent in the brain tumor field.

The second thing we learn from general oncologists is that, of course, we need to have interaction in the field of brain metastasis or meningeal disease, because this is a not-so-rare complication of even successfully treated patients with breast cancer. For example, even in a favorably prognostic triple-positive breast cancer patient brain metastasis can occur, and this is life limiting and also quality-of-life limiting. The same is true for non-small-cell lung cancer. We also have this extended stage III disease where quite a few patients respond very well to the current treatment but several patients develop CNS metastases This is why we need to have a good interaction between departments.

\section{As Director of the National Center} for Tumor Diseases Novel Therapeutics Area, which novel therapeutics in development do you see as having the most potential to translate into clinical benefits for patients?

The most direct evidence at the moment is a positive, controlled trial with a CD95L inhibitor, termed APG101, in conjunction with radiotherapy for recurrent glioblastoma [1]. It shows benefits of this CD95 ligand inhibition together with radiotherapy, compared with radiotherapy alone. I think this is something that is interesting.

More generally speaking, this is showing you that with an interesting and intelligent combination of very old treatments, such as radiotherapy, with a breaking paradigm approach, such as the CD95 ligand inhibition, we may make progress in the treatment of patients. 
From my perspective I expect a new wave of immunotherapy - I believe that there is now new genetic information we are getting on tumors that will reveal not only more targets for small molecules but also targets that are more specifically immunogenic. My hope is for these types of immunotherapy trial, where we test both active immunotherapy with either peptides, cells or RNAs, and at the same time inhibit the immunosuppressive environment in brain tumors. I like that approach. I think the melanoma field and the prostate cancer field are guiding the way in that area with factors targeting the immune system-tumor microenvironment.

Lastly, I still hope for the first of all those targeted therapies to be effective in brain tumors. I think that the trials I am seeing at the moment are increasingly limiting patient entry to certain molecular subtypes and this may have more promise than the one-fits-all approaches that we had until a very short time ago.

What has been the best lesson you have learnt in your career \& how would you advise someone about to specialize in neuro-oncology?

Neuro-oncology makes no real sense without splitting your work into some research parts - because you need to really understand this disease and the molecular basis of the disease - and a dedicated clinical part. It makes sense to see patients. I think it is key to look and ask for their perspective of the disease all through your career.

You need to talk to the patients. For example, when we came up with the elderly trial, this was a question actually asked by a patient: "Why should I receive the same treatment as that of the 30-year-old patient in the waiting room. I'm much older and I have a totally different life perspective." You need to stay in tune with your patient's needs.

What would be your vision for progress in neuro-oncology over the next 10 years?

In the past 18 months there has been so much knowledge generated on the molecular basis of brain tumors - gliomas specifically but also on medulloblastoma and ependymoma. With a little bit more work on that, with partners in the pharmaceutical industry that are acknowledging that we are treating a very heterogeneous group of patients, I think we will make the next step.

Whether and how much immunotherapy plays a role, its my personal view that it will. We will see the first positive immunotherapy trials in the next 10 years and we will probably also see some progress on the stem cell discussion. I'm not seeing a role for the stem cell niche at the moment, but there may be smart ways of at least treating patients with a kind of maintenance treatment. I like this concept of after heavy treatment, to not just wait and see, but instead to continue a type of maintenance treatment. After that, then the ideas and different concepts of those that are working on cancer stem cells may come into the game.

\section{Disclaimer}

The opinions expressed in this interview are those of the interviewee and do not necessarily reflect the views of Future Medicine Ltd.

Financial \& competing interests disclosure

$W$ Wick has received research support or honoraria for lectures from Apogenix, Boehringer Ingelheim, Eli Lilly, MSD and Roche, served on the Steering Committee of the Roche sponsored AVAglio trial. $W$ Wick has no other relevant affiliations or financial involvement with any organization or entity with a financial interest in or financial conflict with the subject matter or materials discussed in the manuscript apart from those disclosed.

No writing assistance was utilized in the production of this manuscript.

\section{Reference}

1 Bendszus M, Debus J, Wick W et al. A Phase II, randomized, open-label, multi-center study of weekly APG101 + reirradiation versus reirradiation in the treatment of patients with recurrent glioblastoma. J. Clin. Oncol. 30, Abstract 3304 (2012). 